\title{
Interpreting physical flows in networks as a communication system
}

\author{
NICOLÁS RUBIDO ${ }^{1, *}$, CELSO GREBOGI $^{2}$ and MURILO S. BAPTISTA ${ }^{2}$ \\ ${ }^{1}$ Universidad de la República, Instituto de Física de la Facultad de Ciencias, Iguá 4225, Montevideo, \\ 11400, Uruguay \\ ${ }^{2}$ University of Aberdeen, King's College, Institute for Complex Systems and Mathematical Biology, \\ AB24 3UE Aberdeen, UK \\ *Corresponding author. E-mail: nrubido@ fisica.edu.uy
}

\begin{abstract}
Information theory concepts and methodologies constitute the background of how communication systems are studied and understood. They are focused mainly on the source-channel-receiver problem and on the asymptotic limits of accuracy and communication rates, which are the classical problems studied by Shannon. However, the impact of information theory on networks (acting as the channel) is just starting. Here, we present an approach to understand how information flows in any connected network. Our approach is based on defining linear conservative flows that travel through the network from single or multiple sources to receivers. With these flows, we define a transition probability matrix that is similar to a Markovian process. Consequently, this framework allows us to have an analytical description of the problem and also to link the topological invariants of the network, such as the node degree, with the information flow and capacity, namely, the maximum amount of information generated by the network for any source-receiver configuration. In particular, our approach is able to deal with information transmission in modular networks (networks containing community structures) or multiplex networks (networks with multiple layers), which are nowadays of paramount importance.
\end{abstract}

Keywords. Complex networks; flow networks; information measures; random walks.

PACS Nos 89.75.Hc; 45.30.+s; 02.50.-r; 41.20-q

\section{Introduction}

The physical universe is ruled by physical laws. These laws are constructed under the principle that any two bodies interact among themselves by the force between them. The interaction level is measured by the force magnitude. In the field of complex systems, an extension of this concept, and widely employed quantity to measure the interaction level between two or more systems, is their synchronisation degree $[1,2]$. Namely, a measure that quantifies the similarity between their behaviour. From the perspective of information theory, similar behaviours correspond to significant information sharing. Namely, knowing what one system is doing allows one to predict with high accuracy what the other system is doing [3]. For example, the mutual information rate was recently proposed as a way to measure how two dynamical systems (or group of systems) coupled in a network are related to each other $[4,5]$, which has also proven useful to infer their structural connection [6] where inferring by synchronisation degree fails. Consequently, the main question that we raise is to understand how strong will two systems or networks interact by analysing them as a communication system.

On the one hand, using dynamical networks to generate encodings, such that the information transmission is enabled, has proven to be an important task in recent years. In this sense, an outstanding achievement is the Computing Reservoir technique [7-9], which is somewhat of an extension of the well-known neural networks. These networks are composed of active elements or nodes. Nevertheless, the information generation/transmission, underlying mechanisms, and practical capabilities of such techniques are not fully understood yet. On the other hand, network structures have recently been used to assess their vulnerability to cascade failures [10-14], and to a lesser extent, their information capabilities $[15,16]$. These works are based on passive elements and discard physical quantities that might be transported through the network, focussing solely on the network's structure. Hence, they are unsuitable to be analysed as a communication system since there is no transmitter or receiver. 
In an effort to bridge this gap, some works have dealt with transportation problems on passive networks, namely, traffic flows, setting routines and rules to transport packages across the network and finding numerically the resulting flows and jamming properties [17-21]. However, aside from power-law behaviours that emerge under particular topologies or under thermodynamic limits, the resulting flows are mathematically untractable and depend on the location of the transmitter and receiver.

Here, we present a different approach to the dynamical networks and to the former transportation networks, specially when queuing packages is possible. We use passive networks and define physical flows on top of their structure by setting electric currents that travel from sources to sinks, i.e., we define a tractable transportation system. Thus, our apporach becomes analytical (even for finite-size systems) and it allows us to quantify how information is transmitted in any complex network and how much information the network can handle under any source-sink configuration, namely, its capacity.

We define the physical flows by interpreting a subset of its nodes to be transmitters (sources), and another subset to be receivers (sinks), as is shown in figure 1 . The information transmitted is derived from these physical flows by encoding them similarly to how it is done when random walks are defined on graphs, except that here we use the network's structure and the flow distribution resulting from the transmitter input and receiver ouput. For example, in the case of random

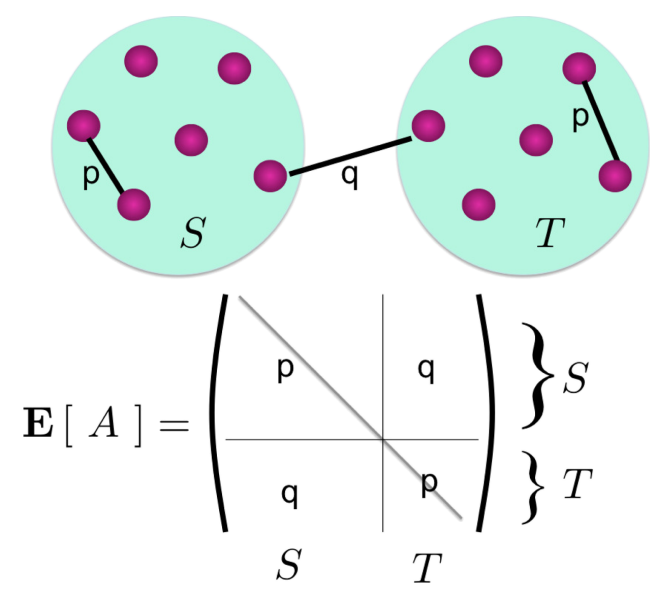

Figure 1. The top panel shows schematically our communication network, where we divide the network in a subset of transmitter [receiver] nodes, $S[T]$, that are connected by edges, $q$. For example, this division can be straightforwardly set in modular networks. The bottom panel shows the corresponding information flow matrix $\mathbf{E}[A]$, which depends on the network's structure given by its adjacency matrix, $A$. walkers in graphs, the voltage potential at the nodes of a resistor network can be linked to the probability of finding the walker at the node [22-25]; however, a voltage is always defined up to an arbitrary reference value. On the contrary, our approach uses the voltage potential differences and the edge weights (resistance) to define the physical flows (currents), thus defining invariant physical quantities. The information flows are derived from these physical flows by setting them as the probability for the random walker to take a jump between sites in the network (see eq. 5). Consequently, our formulation allows us to derive exact and approximate expressions for the resultant information flows for any network and any transmitter-receiver configuration, thereby bridging the gap between single-channel communication systems and multiple-channel communication systems. Moreover, we are able to interpret directly the information transmission capacity of the network with the network invariants, such as the node degree.

\section{Methods and model}

The starting point in our approach is to define and to analytically solve the flows in a conservative transport network, namely, solve the Voltage-Flow (VF) problem in a network. The model for our VF problem is that of an ohmic (passive) circuit [26] with input and output currents, i.e., a resistor network, $\mathcal{G}$, where each edge current, $f_{i j}$, is linearly related to the potential difference, $\Delta V_{i j}$, between the nodes that the edge connects ( $i$ and $j$ ) and its resistance, $R_{i j}$. Specifically,

$f_{i j}=\frac{\Delta V_{i j}}{R_{i j}}$.

The input currents are set in a node subset, $S \subset \mathcal{G}$, and the total input leaves the network at another node subset, $\mathcal{T} \subset \mathcal{G}$, corresponding to the network's output. In other words, a total current $I$ enters the circuit at some nodes and the same amount $I$ leaves the circuit at other nodes, thus defining a conservative-flow network (which means that the total current arriving at any node is equal to the total current leaving the node). Consequently, the values that the edge flows, $f_{i j}$, take in eq. (1) depend on the location of the source and sink nodes and the magnitude of the total input flow, namely, $f_{i j}^{(S, \mathcal{T})}$. Here, without loss of generality, we define a VF problem with $I=1$ and set a single-source/single-sink system (for an extension of the problem to multiple sources and sinks, see, for example, [27]), thus, the edge flow is $f_{i j}^{(s, t)}$.

In particular, the resistor network is derived from the network structure; hence, it is defined from the set 
$\mathcal{G}=\{\mathcal{V}, \mathcal{E}\}$, where $\mathcal{V}$ and $\mathcal{E}$ are the node and edge sets, respectively. We restrict ourselves to symmetric networks by setting the edge weights as $W_{i j} \equiv A_{i j} / R_{i j}$, with $i, j=1, \ldots, N, N$ being the number of nodes in $\mathcal{V}, A_{i j}$ the $i j$-th element of the adjacency matrix, and $R_{i j}$ the edge's resistance. In the cases where the network is unweighted, then $W_{i j}=A_{i j}$. Consequently,

$\mathbf{G} \vec{V}^{(s t)}=\vec{F}^{(s t)}$,

where the upper indexes indicate that the VF problem depends on the location of the source-sink nodes, $s-t, \vec{F}^{(s t)}$ is the flow vector containing the total current at each node (i.e., $\left[\vec{F}^{(s t)}\right]_{i}=\sum_{j} f_{i j}^{(s, t)}=\delta_{i s}-\delta_{i t}$ for $i=1, \ldots, N, \delta_{i j}$ being the Kronecker delta), and $\mathbf{G}$ is the weighted Laplacian matrix whose entries are

$G_{i j}= \begin{cases}\sum_{k=1}^{N} W_{i k} & \text { if } i=j, \\ -W_{i j} & \text { if } i \neq j .\end{cases}$

The solution for the VF problem (eq. (2)) is achieved once the voltages at each node are found from inverting $\mathbf{G}$. However, because $\mathbf{G}$ is a Laplacian matrix, its inverse is ill-defined (its kernel has non-null dimension). Despite this, we can use the Moore-Penrose pseudo inverse matrix to invert $\mathbf{G}$, allowing us to find an exact value for the voltage differences [27-31]. The result is that

$\Delta V_{i j}^{(s, t)}=\sum_{n=2}^{N} \frac{\left(\Delta\left[\vec{\psi}_{n}\right]_{i j}\right)}{\lambda_{n}}\left(\Delta\left[\vec{\psi}_{n}\right]_{s t}\right)$,

where $\vec{\psi}_{n}$ is the $n$-th eigenvector associated to the eigenvalue $\lambda_{n}$ of $\mathbf{G}$ (i.e., $\mathbf{G} \vec{\psi}_{n}=\lambda_{n} \vec{\psi}_{n} \forall n$ ) and $\Delta\left[\vec{\psi}_{n}\right]_{i j} \equiv\left[\vec{\psi}_{n}\right]_{i}-\left[\vec{\psi}_{n}\right]_{j}$ is the $i$ and $j$ eigenvectorcoordinate difference for the $n$-th eigenmode. We note that the first eigenmode, $n=1$, corresponds to $\lambda_{1}=0$ and $\vec{\psi}_{1}=\overrightarrow{1} / \sqrt{N}$, which is the condition that any Laplacian matrix row-sum is null, hence, its kernel has at least dimension 1 [28, 29]. Also, the edge flows, $f_{i j}^{(s, t)}$, are straightforwardly derived from this expression by means of eq. (1). Furthermore, eq. (4) shows how the location of the source and sink nodes modify the voltage difference value by means of the corresponding eigenvector-coordinate difference.

Eq. (4) is the main derivation that allows us to define our information flows approach. It is applicable to any connected weighted graph and is extendible to many sources and sinks with different inputs and outputs, as long as flow conservation is fulfilled. Specifically, we define our information flows from the following flowtransmission probability matrix, $\boldsymbol{\Pi}^{(s, t)}$, with $i j$ entry

$\Pi_{i j}^{(s, t)} \equiv \frac{1}{d_{i}}\left|f_{i j}^{(s, t)}\right|$,

where $d_{i} \equiv \sum_{j}^{N}\left|f_{i j}^{(s, t)}\right|$, which is twice the total inflow (by conservation, it also means twice the total outflow) at node $i$ because $f_{i j}^{(s, t)}=-f_{j i}^{(s, t)}$. By constructing this transition matrix (eq. (5)), we are detaching ourselves from random walks $(R W)$ on graphs, which solely depend on the network's structure, and we are including the functionality of the network as a communication channel.

An RW on a graph is defined by a transition probability that depends on the graph's structure, namely, $\Pi_{i j}^{(\mathrm{RW})} \equiv W_{i j} / d_{i}$, where $W_{i j}$ is the $i j$ edge's weight and $d_{i}$ the weighted degree. This probability matrix is asymmetric $\left(\Pi_{i j}^{(\mathrm{RW})} \neq \Pi_{j i}^{(\mathrm{RW})}\right)$ but it fulfils detailed balance, i.e., $p_{i} \Pi_{i j}^{(\mathrm{RW})}=p_{j} \Pi_{j i}^{(\mathrm{RW})}$ for every node $i$ and $j$ and probability distribution $\vec{p}[32,33]$. Hence, it determines a Markov process: $\vec{p}(n)=\vec{p}(0)\left[\Pi^{(\mathrm{RW})}\right]^{n}, \forall n \in$ $\mathbb{N}^{+}$. Consequently, it converges to a unique stationary probability distribution (SPD), regardless of the initial condition.

\section{Results and discussion}

Our approach to define information flows (i.e., the flowtransmission probability matrix, $\mathbf{\Pi}^{(s, t)}$ ), allows us to include the network's structure and function into the problem. In other words, our random walker takes into account the network's structure at each node (e.g., how likely is to jump to any of its neighbours given the number of neighbours it has) and also the net current strength at the node $\left(\sum_{j}^{N}\left|f_{i j}^{(s, t)}\right|\right)$, which is determined by the locations of $s$ and $t$. In this way, we force a direction for the random walker to follow the paths that take it from the source (transmitter) to the sink (receiver). Moreover, since our formulation follows the main mathematical properties of the classical RW on graphs [32,33], our $\boldsymbol{\Pi}^{(s, t)}$ converges to a SPD as well, regardless of the initial conditions, namely, regardless of the initial probability distribution (which is independent of $s$ and $t$ ). Specifically, $\vec{p}(n)^{(s, t)}=\vec{p}(0) \boldsymbol{\Pi}^{(s, t)^{n}} \stackrel{n}{\rightarrow} \vec{\mu}^{(s, t)}$, where $\vec{\mu}^{(s, t)}$ and $\vec{p}(n)^{(s, t)}$ inherit the dependence on $s$ and $t$ because of our flow-transition matrix definition in eq. (5).

We note that in the RWs on unweighted graphs, the SPD is $\mu_{i}^{(R W)}=k_{i} / \sum_{i} k_{i}$ [32], where $k_{i}=\sum_{j=1}^{N} A_{i j}$ is the $i$-th node degree. Hence, the RW SPD is directly 
dependent on the degree distribution of the network alone. On the other hand, our SPD, $\vec{\mu}^{(s, t)}$, depends on where the transmitter and receiver are located as well as on the flow-weighted degree distribution (i.e., it depends on $\left.d_{i}=\sum_{j}^{N}\left|f_{i j}^{(s, t)}\right|\right)$. Nevertheless, analogously to the RW, our SPD can be derived from our flow-transition probability matrix (FTPM), $\mathbf{\Pi}^{(s, t)}$, using standard techniques $[32,33]$ as we do in what follows.

The reason to obtain a unique SPD is that our FTPM is a stochastic matrix with positive entries that has unit row-sums $\left(\sum_{j} \Pi_{i j}^{(s, t)}=1 \forall i\right)$, as can be directly verified from eq. (5). Consequently, its eigenvalue spectrum is bounded in the complex plane by the disc of unit radius, as the Gershgorin theorem predicts [29]. Moreover, this means that there is a non-degenerate maximum-valued eigenvalue $\alpha_{1}=1$, which we arbitrarily set to the first eigenmode of the FTPM, that is non-vanishing. In what follows, we assume that the FTPM is irreducible and aperiodic; thus, $\alpha_{1}$ is non-degenerate and its right eigenvector set, $\mathbf{P}=\left\{\vec{v}_{1}, \ldots, \vec{v}_{N}\right\}$, is orthonormal (i.e., $\left.\vec{v}_{i} \cdot \vec{v}_{j}=\delta_{i j} \forall i, j\right)[32,33]$. Since $\boldsymbol{\Pi}^{(s, t)} \vec{v}_{n}=\alpha_{n} \vec{v}_{n}$ for every eigenmode, the stationary FTPM is

$\boldsymbol{\Pi}^{(s, t)^{n}}=\mathbf{P} \Lambda^{n} \mathbf{P}^{-1} \stackrel{n}{\rightarrow} \mathbf{P}\left[\begin{array}{ccc}1 & 0 & \cdots \\ 0 & 0 & \cdots \\ 0 & 0 & \ddots\end{array}\right] \mathbf{P}^{-1}$,

where $\boldsymbol{\Lambda}$ is the diagonal matrix containing the eigenvalues of the FTPM (i.e., $\boldsymbol{\Lambda}=\left\{1, \alpha_{2}, \ldots, \alpha_{N}\right\}$ ) and $\mathbf{P}$ is the matrix containing in each column the right eigenvectors of the FTPM, which depend on $s$ and $t$. This means that the limit process selects the product between P's first column, that is $\vec{v}_{1}=\overrightarrow{1} / \sqrt{N}$ (as with the Laplacian matrix), and $\mathbf{P}^{-1}$ 's first row. Thus, the stationary FTPM entries are given by

$\left[\mathbf{\Pi}^{(s, t)^{n}}\right]_{i j} \stackrel{n}{\rightarrow} \frac{\left[\mathbf{P}^{-1}\right]_{1 j}}{\sqrt{N}}, \forall i=1, \ldots, N$

The first result from taking our approach and define flows on the network, is that we are able to derive an expression for our SPD, $\vec{\mu}^{(s, t)}$, in terms of eq. (7). Specifically, for any initial distribution $\vec{\mu}_{0}$ converges to

$\vec{\mu}_{0} \boldsymbol{\Pi}^{(s, t)^{n}} \stackrel{n}{\rightarrow}\left(\frac{\left[\mathbf{P}^{-1}\right]_{11}}{\sqrt{N}}, \frac{\left[\mathbf{P}^{-1}\right]_{12}}{\sqrt{N}}, \ldots\right)=\vec{\mu}^{(s, t)}$.

Consequently, we can compute the amount of information encoded in the network, $H^{(s, t)}$, due to the flows in the particular $s-t$ configuration by

$H^{(s, t)}=-\sum_{i=1}^{N} \mu_{i}^{(s, t)} \log \left[\mu_{i}^{(s, t)}\right]$.
We note that the expression for $\vec{\mu}^{(s, t)}$ is also the one for the left eigenvector of the FTPM maximum-valued eigenvalue, because the Markov process defined by the FTPM converges when $\vec{\mu}^{(s, t)}=\vec{\mu}^{(s, t)} \Pi^{(s, t)}$. As a left eigenvector, it has to have unit $L_{1}$-norm, i.e., $\left\|\vec{\mu}^{(s, t)}\right\|_{1}$ $=1=\sum_{i}\left|\mu_{i}^{(s, t)}\right|$, because they constitute probability distributions, contrary to the right eigenvectors of $\boldsymbol{\Pi}^{(s, t)}$ which have unit $L_{2}$-norm (also known as the Euclidean norm).

The network's maximum information, namely, its information capacity $C$, is achieved when searching for the maximum $H^{(s, t)}$ after changing $s$ and $t$ 's location around the network, i.e.,

$C \equiv \max _{s, t \in \mathcal{G}}\left\{H^{(s, t)}\right\}$

Because we have an analytical expression for the flows in the network (eq. 4), we can compute the information flows directly (eq. 5), and thus, we are presenting an efficient approach to compute $C$ straightforwardly.

The second result comes from the information shared in the network between source and sink at any given time $n$, i.e., the mutual information (MI). In particular, we find that under the SPD conditions, the network's MI is null. In general, the mutual information of a given $s-t$ configuration at an instant $n$ is defined as

$I_{(s, t)}(n) \equiv \sum_{i, j}^{N} p_{i, j}^{(s, t)}(n) \log \left[\frac{p_{i, j}^{(s, t)}(n)}{p_{i}(n)^{(s, t)} p_{j}(n)^{(s, t)}}\right]$,

where $p_{i, j}^{(s, t)}(n)$ is the joint probability to find node $i$ and $j$ having the same event at time $n$, which in our case, corresponds to the same information flows determined by eq. (5). In order to understand this, we recall that a joint probability can always be defined from the probability distribution and the transition probabilities by

$p_{i, j}^{(s, t)}(n) \equiv p_{i}(n)^{(s, t)}\left[\boldsymbol{\Pi}^{(s, t)}\right]_{i j}$.

This expression also shows that the joint probability always depends on the location of $s$ and $t$, contrary to the initial probabilities $p_{i}(0)$. According to eq. (12), when $n \rightarrow \infty$ we retrieve the SPD, $p_{i}(n) \mapsto \mu_{i}^{(s, t)}$, and the stationary transition probability, $\left[\boldsymbol{\Pi}^{(s, t)}\right]_{i j} \mapsto \mu_{j}^{(s, t)}$ (eq. 7). Thus, the joint probability becomes decoupled. In other words, when the information flows become stationary, the probabilities at each node are independent quantities which only depend on the location of the source and sink and the network structure. 
As a final result, and to illustrate our approach, we apply eqs (5-9) to an unweighted modular network with $N=100$ nodes, two identical modules with 50 nodes each, identical edge density $\rho=0.5$, and Erdös-Rényi (ER) network characteristics [34]. We place 10 extra links randomly between the modules to interconnect them.

In order to contrast our results with that of a classical RW, we first show in figure 2 the RW transition probability matrix, $\Pi^{(\mathrm{RW})}$, (top panel) and the corresponding SPD, $\vec{\mu}^{(\mathrm{RW})}$ (bottom panel). From the top panel, it can be seen that the walker is able to transit through the network only according to the network's structure, as each transition is defined by $\Pi_{i j}^{(\mathrm{RW})}$ $\equiv A_{i j} / k_{i}$. Moreover, the SPD is the vector of node degrees, $\vec{k}$, divided by the total edge number, which in this case, is $\vec{\mu}^{(\mathrm{RW})}=\vec{k} / 2492$. We recall that the expected edge number, $E[M]$, for an ER network of size $N$ is: $E[M]=\rho N(N-1) / 2$, which in our case corresponds to $E[M]=612.5$ for each module. Thus, taking into account both modules plus the extra inter-links, the total expected edges are $2(2 M)+10=2460$, which is close to the exact value in our case of study, namely, $2492=\sum_{i} k_{i}$.

On the other hand, we set a source (transmitter) in one module, $s=46$, and a sink (receiver), $t=90$, in the other module. This defines a voltage difference between every node in the network (eq. 4). Hence, the information flows have a transition matrix that allows the walker to jump freely between modules,

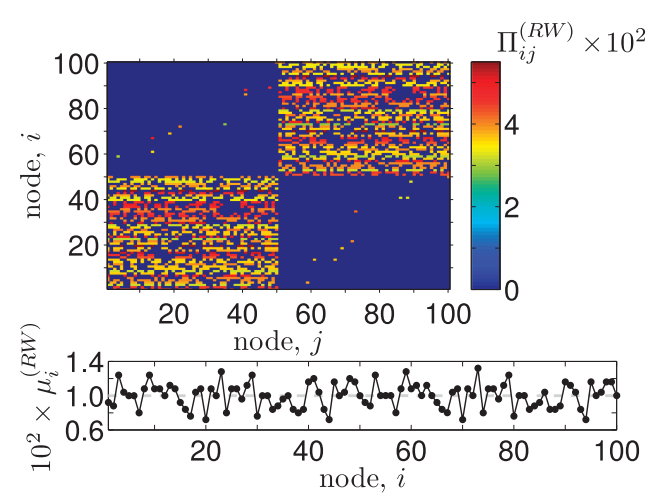

Figure 2. Transition probability matrix (TPM) $\boldsymbol{\Pi}^{(\mathrm{RW})}$ (top panel), and stationary probability distribution (SPD) $\vec{\mu}^{(\mathrm{RW})}$ (bottom panel), for a random walk in a two-module network with $N=10^{2}$ nodes. The two modules are identical, have an Erdös-Rényi topology (namely, they are random) with 50 nodes and a 0.5 edge density, and are interconnected by 10 edges. The TPM entries are represented by the colourcode. Both TPM and SPD are multiplied by $N$ to improve clarity.

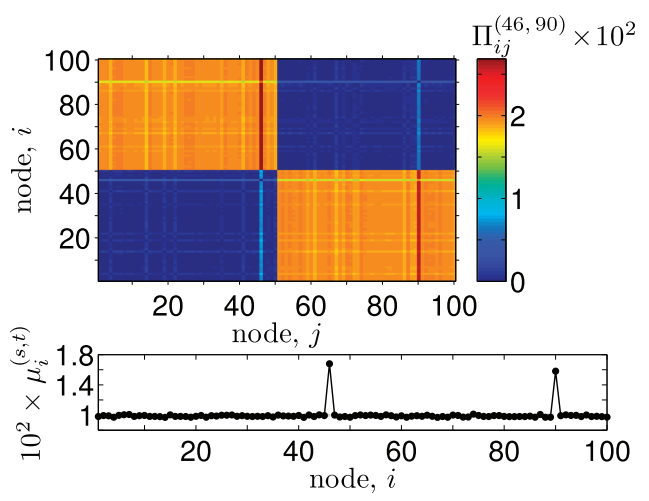

Figure 3. Flow-transition probability matrix (FTPM) $\mathbf{\Pi}^{(s, t)}$ (top panel), and stationary probability distribution (SPD) $\vec{\mu}^{(s, t)}$ (bottom panel), for a source $s$, sink $t$, configuration in the same network as figure 2. Colour codes and normalisation follow the same criteria as in figure 2 .

as is seen in figure 3. Moreover, we can see that the probabilities to jump between modules are higher than the inner jumps, which is the opposite situation to that in the RW. Also, we can directly distinguish the importance of source and sink nodes in the FTPM and in the SPD, because both have higher values than the rest of the nodes.

For this $s$ - $t$ flow configuration, the information generated is comparable with the RW, as we verify with eq. (9), resulting in $H^{(\mathrm{RW})} \simeq 6.63$ bits and $H^{(46,90)} \simeq 6.64$ bits. However, if we place the transmitter and the receiver in the same module, most FTPM values become nearly null, with the exception of those that correspond to the paths in the module joining the source with the sink. Consequently, the information decreases. For example, the configuration with $s=46$ and $t=30$ results in $H^{(46,30)} \simeq 5.74$ bits. This means that the network's information capacity (eq. 10) is influenced mainly by the number of modules in the network, with the maximum achieved when source and sink are set the furthest apart. Similar results are expected for multilayered networks, since each layer can be thought of as a different module.

\section{Conclusions}

In this work we present a rather novel approach to tackle the problem of information transmission in networks. We define a circuit-like network based on the network's structure, set a source [sink] node that inputs [outputs] a constant flow, and solve the voltage-flow problem for every edge in the network. By doing this, we are able to define a flow-transition probability matrix, similar to how it is done with random walks in graphs, but instead of using solely the network's 
structure, we use the resultant flows. The reason behind defining the source-sink nodes is that it allows us to interpret the problem as a transmitter-receiver problem whose communication channel has multiple paths. Consequently, with our approach, we have a tractable way to understand how the information is transmitted across the network.

In particular, we see that modular networks have an information capacity that increases with its modularity, namely, with the number of communities in the network. In general, we see that the further apart the transmitter and receiver are, the higher the information that is generated in the network. This conclusion leads us to think that modular networks have higher information capacities than non-modular networks due to the bottlenecks that inter-links constitute for the flows. However, we note that further research in this line is needed in order to have sound conclusions. Moreover, we expect similar results to hold also for multi-layered or multiplex networks, where source and sink should be placed in different layers in order to increase the overall network capacity.

In general, the paper lays down a new theoretical framework to interpret how physical flows in a flow network can be used to encode and transmit information in a communication system based on networks or multiplex networks, where one node or network has the role of a transmitter and another node or network has the role of a receiver. We demonstrate how to calculate information capacities for the whole transmitterreceiver multiplex networks, and discuss how mutual information can be calculated in this framework. Hence, we have put forward a method to interpret mutual information in this communication network as a quantity that measures the amount of information that is exchanged in the whole transmitter-receiver network at a given state and time. Since the probabilities we define from the physical flows have Markovian memoryless properties, the considered mutual information is null when the system reaches the steady-state equilibrium. This, however, does not mean that the proposed system cannot be used to transmit information, only that another interpretation of the mutual information should be used to transform this theoretical framework into a practical communication system. Future work should demonstrate that in order for the network to transmit all the information available (whose upper bound is provided by the information capacity of the network), the definition of the mutual information (reflecting the way information is actually transmitted) should split the probability set and the transition probability matrix into two sets, one set related to the transmitter at a given time, and another set related to the receiver at another time.

\section{Acknowledgements}

NR acknowledges the support of PEDECIBA, Uruguay. CG and MSB thank the Scottish University Physics Alliance (SUPA) support. MSB also acknowledges the support of EPSRC grant Ref. EP/I032606/1.

\section{References}

[1] A Pikovsky, M Rosenblum and J Kurths, Synchronization: A Universal Concept in Nonlinear Sciences (Cambridge university press, Cambridge, 2003)

[2] S Strogatz, Sync: The emerging science of spontaneous order (Hyperion, New York, 2003)

[3] C E Shannon, A mathematical theory of communication (Bell System Technical Journal, 27, 1948)

[4] M S Baptista, J X de Carvalho and M S Hussein, PLoS ONE 3(10), e3479 (2008)

[5] M S Baptista and J Kurths, Phys. Rev. E 77, 026205 (2008)

[6] E Bianco-Martínez, N Rubido, C G Antonopoulos and M S Baptista, Chaos 26, 043102 (2016)

[7] H Jaeger and H Haas, Science 304(5667), 78 (2004)

[8] M Hermans and B Schrauwen, Neural Networks 23(3), 341 (2010)

[9] Y Paquot, et al., Sci. Rep. 2 (2012)

[10] I Simonsen, L Buzna, K Peters, S Bornholdt and D Helbing, Phys. Rev. Lett. 100, 218701 (2008)

[11] W X Wang and Y C Lai, Phys. Rev. E 80(3), 036109 (2009)

[12] S V Buldyrev, R Parshani, G Paul, H E Stanley and S Havlin, Nature 464(7291), 1025 (2010)

[13] A Cardillo, et al., Eur. Phys. J. Special Topics 215, 23 (2013)

[14] S Pahwa, C Scoglio and A Scala, Sci. Rep. 4, 3694 (2014)

[15] R V Solé and S Valverde, Complex Networks: Lecture Notes in Physics (Springer, 2004) Vol. 650, pp. 189

[16] A Nematzadeh, E Ferrara, A Flammini and Y Y Ahn, Phys. Rev. Lett. 113(8), 088701 (2014)

[17] B Tadi $\not$ and V Priezzhev, Eur. Phys. J. B 30, 143 (2002)

[18] B Tadiф, G J Rodgers and S Thurner, Int. J. Bif. Chaos 17(7), 2363 (2007)

[19] Y-H Chen, et al., Phys. Rev. E 81, 066105 (2010)

[20] R Carvalho, L Buzna, W Just, D Helbing and D K Arrowsmith, Phys. Rev. E 85(4), 046101 (2012)

[21] Z-Y Jiang, J-F Ma and X Jing, Physica A 422, 181 (2015)

[22] P Tetali, J. Theor. Prob. 4(1), 101 (1991)

[23] P G D J L Snell and P Doyle, FSF (2000)

[24] M E J Newman and M Girvan, Phys. Rev. E 69, 026113 (2004)

[25] N Rubido, C Grebogi and M S Baptista, Europhys. Lett. 101, 68001 (2013) 
[26] G Kirchhoff, Vorlesungen über Mechanik (Wien, Wilhelm, Ed. 1864-1928)

[27] N Rubido, C Grebogi and M S Baptista, Phys. Rev. E 89, 012801 (2014)

[28] B Bollobás, Modern Graph Theory (Springer-Verlag, NY, 1998)

[29] Fan R K Chung, Spectral Graph Theory (Am. Math. Soc. and CBMS 92, 1997)
[30] F Y Wu, J. Phys. A: Math. Gen. 37, 6653 (2004)

[31] A Ghosh, S Boyd and A Saberi, SIAM Rev. 50(1), 37 (2008)

[32] F Göbel and A A Jagers, Stochastic processes and their applications 2(4), 311 (1974)

[33] L Lovász, "Random Walks on Graphs: A Survey”, Combinatorics, Paul Erdös is eighty, Vol. 2, pp. 1-46 (1993)

[34] P Erdös and A Rényi, Publ. Math. 6, 290 (1959) 